\title{
Critical appraisal of clinicopathological features of anaplastic carcinoma small intestine - A rare case report
}

\author{
Nangia Anita, Puri Vandana, Sehgal Shivali \\ Department of Pathology, Lady Hardinge Medical College \& associated hospitals, New Delhi, India.
}

Correspondence: Vandana Puri. Address: Department of Pathology, Lady Hardinge Medical College, New Delhi, India. Telephone: 91-011-981-1952-205. E-mail: drvandanapuri@gmail.com

Received: April 15, 2012

Accepted: May 16, 2012

Published: June 1, 2012

DOI : $10.5430 /$ jst.v2n3p71

URL: http://dx.doi.org/10.5430/jst.v2n3p71

\begin{abstract}
Anaplastic carcinoma is a rare variant of small intestine adenocarcinoma with few cases reported in literature. We report a case of 46 year old male who presented with an endophytic tumor in ileum extending through full thickness of intestinal wall, with invasion into mesenteric fat. Microscopically, the tumor showed a biphasic growth pattern unlike conventional adenocarcinoma. The present case discusses the histomorphology of anaplastic carcinoma of small intestine, its histochemical and immunohistochemical profile and the differential diagnosis of this rare but highly aggressive pathological entity.
\end{abstract}

\section{Key words}

Anaplastic carcinoma, Intestine, Immunohistochemistry

\section{Introduction}

Carcinomas of the small intestine are very rare, with an incidence of 0.5 to 0.8 per 100,000 population per year ${ }^{[1]}$. Anaplastic carcinoma is an extremely rare variant of small intestine adenocarcinoma that arises most frequently in ileum and distal jejunum ${ }^{[2]}$. Identification of this rare variant is essential as this tumor follows more aggressive clinical course with high mortality as compared to conventional adenocarcinoma of small intestine. The aim of the present case is to discuss the pathology of anaplastic carcinoma small intestine, its histochemical and immunohistochemical profile and the differential diagnosis of this rare entity.

\section{Case presentation}

A 46 year old male presented with ahistory of coffee coloured emesis and intermittent lower abdominal pain. Ultrasound abdomen and CT scan of abdomen showed marked thickening of intestinal wall with mesenteric lymphadenopathy. An exploratory laparotomy was performed which revealed an endophytic tumor measuring $17 \mathrm{~cm} \times 8 \mathrm{~cm} \times 10 \mathrm{~cm}$ extending through full thickness wall of ileum, with invasion into mesenteric fat (Figure 1). On serial sections, the tumor was seen perforating into the ileal lumen with perforation measuring $4 \mathrm{~cm}$ in diameter. The patient underwent complete ileal 
resection. However he succumbed to disease three weeks after the surgery. On microscopic examination, the tumor showed a variable histological pattern. Most of the areas had round to oval to polygonal cells arranged in sheets with focally dissociated cells. The tumor cells had abundant eosinophilic cytoplasm. The nuclei were round to oval with clumped chromatin and 0-2 prominent nuclei (Figure 2). In some areas there was an attempt to gland formation with presence of intra and extracellular mucin (Alcian blue positive at $\mathrm{pH}$ 2.5). There was a sparse and focal submucosal spindle cell component. The spindle cells were elongated with cytoplasmic and nuclear morphology similar to the more abundant round cells. The tumor cells were seen infiltrating and destroying the smooth muscle of muscularis propria. Five lymph nodes were dissected from the mesentry, all of them showed presence of tumor deposits. In addition 6 nodular tumor deposits were also found within the mesentry. Immunohistochemistry showed diffuse positivity for both cytokeratin (Pan-CK and CK-20) and vimentin in all tumor cells, in all stages of differentiation (Figure $3 \&$ 4). Focal positivity for epithelial membrane antigen (EMA) was also seen. However LCA, CD30, CD10, Alk-1, S-100, CD117, CEA, SMA, HMB-45 and CD34 were all found to be negative

Figure 1. Gross photograph showing endophytic tumor measuring $17 \mathrm{~cm} \times 8 \mathrm{~cm}$ $\times 10 \mathrm{~cm}$ extending through the full

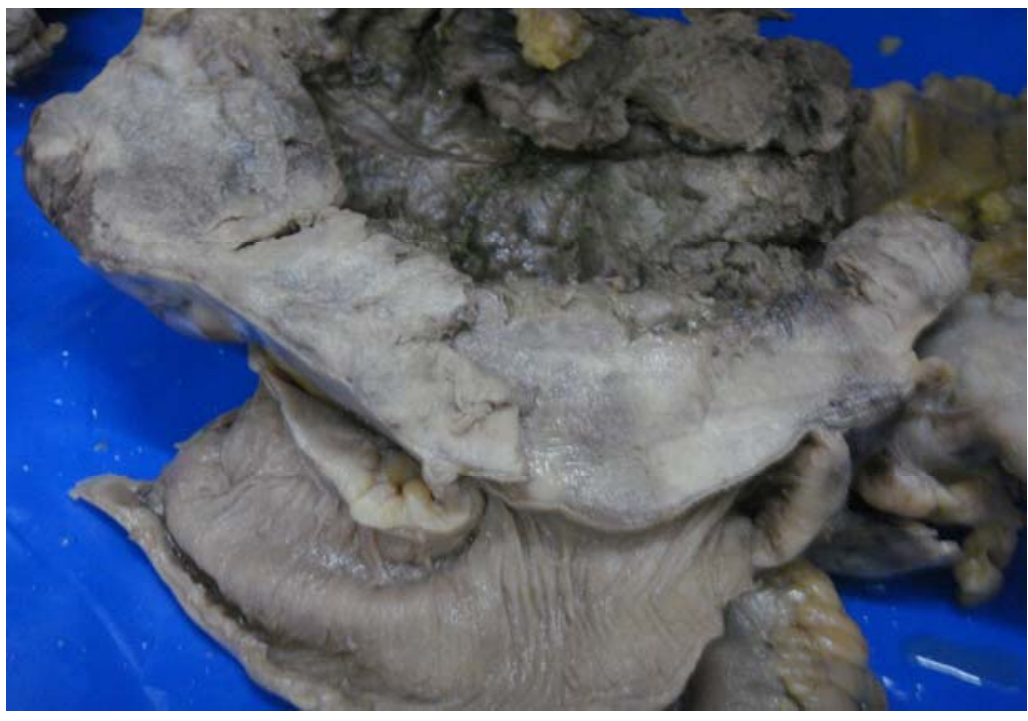
thickness of the wall of ileum

Figure 2. Microphotograph showing tumor cells arranged in sheets with tumor cells having vesicular nuclei and prominent nucleoli. Multinucleated tumor cells are also seen (H\&E 400X)

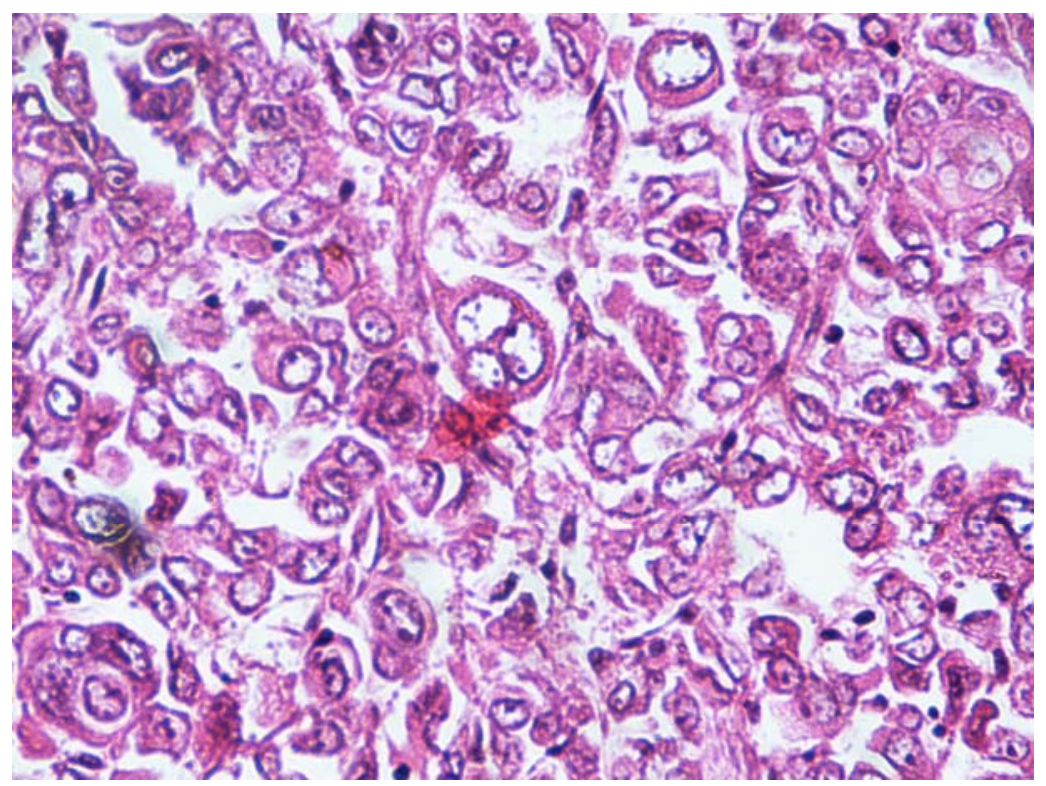


Figure 3. Microphotograph showing CK (pan-CK) positivity in tumor cells

Figure 4. Microphotograph showing vimentin positivity in tumor cells
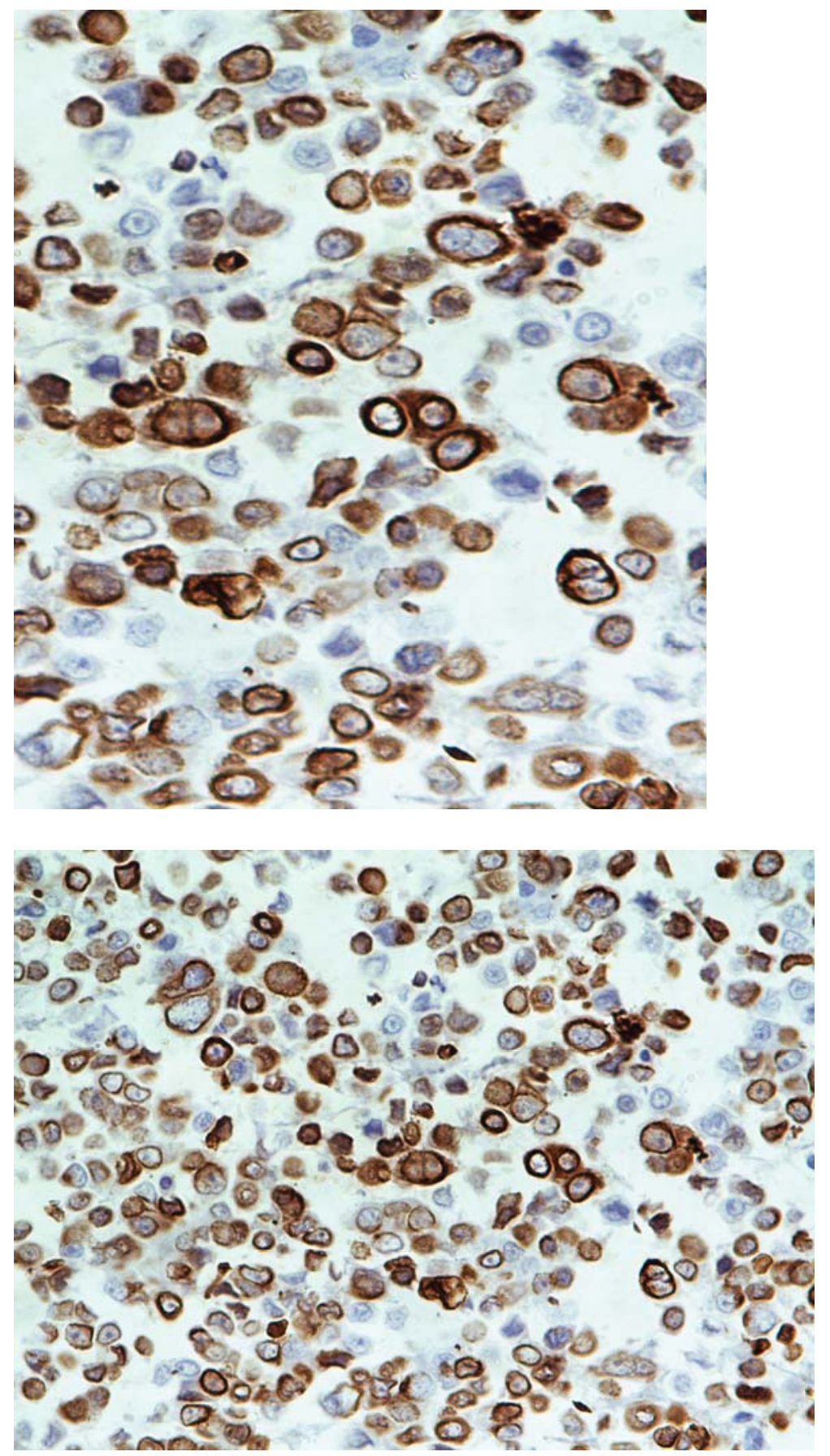

\section{Discussion}

Anaplastic carcinomas of small intestine are rare with only 18 cases reported till date ${ }^{[3]}$. Mean age of presentation is 58 years (Range, 38-81 years) with a slight male predominance, M: F ratio 1.5:1 ${ }^{[4]}$. The most common location for these tumors is the ileum followed by jujenum and rarely duodenum with most presenting as endophytic tumors with central ulceration $^{[4]}$. On routine histological examination, anaplastic carcinoma may appear biphasic or monophasic with a mixture of epithelial and mesenchymal cells ${ }^{[4]}$. The epithelial component may be arranged in sheets, clusters or glands like structures. The mesenchymal component consists of spindle cells with tapering cytoplasm. In both morphologically different areas, nuclei are round to oval vesicular with prominent nucleoli. Foci of anaplasia and tumor giant cells may be 
seen. Sometimes anaplastic cells may predominate to the exclusion of spindle cell component. Such tumors have been called pleomorphic giant cell carcinoma ${ }^{[5]}$. Our case showed a predominantly epithelial component with focal spindle cell areas. On histochemical stains, most small bowel anaplastic carcinomas are negative for mucin however Robey - Cafferty et al. have demonstrated positivity for intracellular mucin in all the six cases they reported ${ }^{[1]}$. Our case showed similar findings. Immunohistochemical, anaplastic carcinomas showed positivity for CK ( $75 \%$ of cases), focal positivity for EMA and focal or diffuse positivity for vimentin (90\% of cases) ${ }^{[4]}$. Typically conventional small intestinal and colorectal adenocarcinomas are positive for CK20 and negative for CK7 ${ }^{[6]}$. Our case also showed CK 20 positivity and negativity for CK7, thereby confirming the primary origin of the tumor, ruling out possibility of metastatic carcinoma to intestine.

Differential diagnosis of anaplastic carcinomas of small intestine includes anaplastic large cell lymphoma (ALCL), epithelioid gastrointestinal stromal tumor (GIST) and metastatic carcinomas as these tumors also showed round to oval to polygonal cells arranged in sheets with vesicular nuclei and prominent nuclei. Multinucleation can also be seen. Hence use of special stains for mucin and a wide immunohistochemical panel becomes essential for arriving at correct diagnosis. ALCL shows positivity for LCA, CD30 and Alk-1. No epithelial dysplasia or mucin is seen. Our case was also negative for LCA, CD30 and Alk-1 with evidence of alcian blue positivity at pH2.5 in mucinous areas of the tumor. GISTs are negative for CK and positive for CD117 and CD34. Our case showed CK positivity and negativity for CD117 and CD34. Positivity for CK20, negativity for CK7 (As in our case) and presence of epithelial dysplasia in the ileal mucosa confirms the presence of primary tumor and rules out metastases to intestine.

To summarize anaplastic carcinomas represent an extremely rare variant of adenocarcinoma small intestine, with aggressive clinical course and extensive local spread. Despite treatment by optimized surgical procedures, patients who have undergone complete tumor resection continue to have a poor prognosis with 3 year survival rate of $10.8 \%$ and median survival time of 5 months ${ }^{[3]}$. Most of the patients with anaplastic carcinoma are in their late stages and presentation with obstructive symptomalogy and perforation is common. Unlike lymphomas and GISTs, its closest differentials, which are amenable to chemotherapy and imitianib respectively, anaplastic carcinomas are best treated by complete surgical resection with chemotherapy and radiotherapy not having any significant effect on the rate of survival. No specific mutations have been detected in the tumor which would allow development of specific targeted therapies. Hence clinical awareness of this entity is essential for early diagnosis and appropriate treatment which will go a long way in reducing disease morbidity and mortality ${ }^{[3]}$.

This case report highlights the histomorphological features of this rare and unusual variant of intestinal adenocarcinoma. Use of immunohistochemistry is essential to arrive at an accurate diagnosis as the tumor histomorphologically mimics other diseases as discussed above. The tumor has highly aggressive behaviour and therefore providing early and optimal clinical management is essential for improving prognosis and survival.

\section{Conflict of interest}

The author declares that there is no conflict of interest statement.

\section{References}

[1] Robey-Cafferty SS, Silva EG, Cleary KR. Anaplastic and sarcomatoid carcinoma of the small intestine: A clinicopathologic study. Hum Pathol 1989; 20: 858-63. http://dx.doi.org/10.1016/0046-8177(89)90097-X

[2] Agrawal S, Trivedi MH, Lukens FJ, Moon C, Ingram EA, Barthel JS. Anaplastic and sarcomatoid carcinoma of the small intestine: An unusual tumor. J Clin Gastroenterol 1999; 29: 99-101. PMid:10405244 http://dx.doi.org/10.1097/00004836-199907000-00025

[3] Namikawa T, Hanazaki K. Clinical analysis of primary anaplastic carcinoma of the small intestine. World J Gastroenterol 2009; 15: 526-30. PMid:19195053 http://dx.doi.org/10.3748/wjg.15.526

[4] Reid-Nicholson M, Idrees M, Perino G, Hytiroglou P. Sarcomatoid carcinoma of the small intestine: A case report and review of the literature. Arch Pathol Lab Med. 2004; 128: 918-21. PMid:15270605

[5] Bak M, Teglbjaerg PS. Pleomorphic (giant cell) carcinoma of the intestine. An immunohistochemical and electron microscopic study. Cancer. 1989; 64: 2557-64. http://dx.doi.org/10.1002/1097-0142(19891215)64:12<2557::AID-CNCR2820641225>3.0.CO;2-G

[6] Rubin BP, Skarin AT, Pisick E, Rizk M, Salgia R. Use of cytokeratins 7 and 20 in determining the origin of metastatic carcinoma of unknown primary, with special emphasis on lung cancer. Eur J Cancer Prev. 2001; 10: 77-82. PMid:11263595 http://dx.doi.org/10.1097/00008469-200102000-00009 